\title{
Therapeutic hysteroscopy in an outpatient office-based setting compared to conventional inpatient treatment: superior? a cohort study
}

\author{
Roos M. Smits ${ }^{1}$ - Nienke P.M. Kuijsters ${ }^{1,2}$ • Loes Braam ${ }^{3}$ - Huib A. A. M. van Vliet $^{1}$ • \\ Benedictus C. Schoot ${ }^{1,4}$
}

Received: 2 March 2016 / Accepted: 17 August 2016 / Published online: 2 September 2016

(C) Springer-Verlag Berlin Heidelberg 2016

\begin{abstract}
Since the introduction of smaller instruments, hysteroscopy is increasingly performed in an office-based setting. The aim of this cohort study was to compare operative hysteroscopy in an office-based setting with inpatient procedures to evaluate differences in procedure and analgesia-related parameters. All office-based hysteroscopic procedures during February 2014 to October 2015 were entered for analysis. Included were morcellation of fibroids, polyps and pregnancy remnants, synechiolysis, diagnostic hysteroscopy, and endometrial ablation. Comparative cases of patients undergoing hysteroscopic surgery in the operating room were searched during the years prior to initiation of the office-based setting (2012 and 2013). During the outpatient surgical procedures, patients were moderate to deeply sedated with propofol and alfentanyl. Two groups of 129 patients were analysed. Median operation time was significantly shorter in the office-based group (11 min [range 1-37]) compared to the operating room group (20 min [range 2-73], $p<0.01$ ). Median admission time was also shorter in the office-based group (135 min [range 60-150] versus $455 \mathrm{~min}$ [range 240-2865]
\end{abstract}

Roos M. Smits

roos.smits@ catharinaziekenhuis.nl

1 Department of Obstetrics and Gynaecology, Catharina Hospital, P.O. Box 1350, 5623, EJ Eindhoven, the Netherlands

2 Department of Electrical Engineering, University of Technology Eindhoven, Eindhoven, the Netherlands

3 Department of Anaesthesiology, Catharina Ziekenhuis, Eindhoven, the Netherlands

4 Department of Obstetrics and Gynaecology, UZ Gent, Ghent, Belgium $(p<0.01))$. The number of incomplete procedures was similar ( $3.9 \%$ versus $2.3 \%, p=0.473$ ). No significant difference in surgical or anaesthesiology complications was observed. Overall complication rate was $4.7 \%$ in the office-based setting and $3.9 \%$ in the operating room setting. Financial analysis showed that procedures in an office-based setting are at least half of the costs as compared to a clinical setting. Officebased hysteroscopic procedures under procedural sedation and analgesia demonstrate a low complication rate as well as shorter operation and admission time compared to outpatient procedures. Office-based hysteroscopic procedures showed lower healthcare costs.

Keywords Office-based hysteroscopy · Office-based hysteroscopic surgery $\cdot$ Procedural sedation and analgesia . Outpatient treatment $\cdot$ Hysteroscopy

\section{Background}

Hysteroscopy is commonly used to diagnose and treat intrauterine abnormalities. The introduction of new hysteroscopic techniques has led to less pain, shorter hospital stay and quicker return to daily activities [1]. Initially, hysteroscopic procedures were performed in operating theatres under general or loco-regional anaesthesia. These anaesthesia methods are associated with high costs and a long recovery time although effective surgery time is rather short. Due to the development of smaller instruments and subsequent reduced need of analgesics, hysteroscopy is increasingly performed as an officebased procedure. Moreover, recent newly introduced devices (such as the hysteroscopic morcellator, second-generation ablation techniques) that reduced procedure time and pain, supported the choice for surgery in an outpatient setting $[2,3]$. The fear of patient discomfort delayed implementation of 
office-based therapeutic procedures [4]. Already a decade ago, cost benefit analysis was in favour of outpatient office-based procedures compared to inpatient treatment $[5,6]$.

Since February 2014, therapeutic hysteroscopy under procedural sedation and analgesia (PSA) is performed in an office-based setting in the Catharina Hospital Eindhoven, the Netherlands on a regular basis. The aim of this cohort study was to evaluate possible differences in procedural and analgesia-related parameters comparing a cohort of patients who underwent operative hysteroscopy in an office-based setting (OBS) with a cohort of patients undergoing procedures in an operation room setting (ORS).

\section{Methods and materials}

Data of all consecutive office-based hysteroscopic procedures during the period February 2014 to October 2015 were included for analysis. Procedure type and exclusion criteria are shown in Table 1. An anaesthesiologist or sedation specialist screened all patients preoperatively. All patients underwent preoperative fasting. Comparative cases of patients undergoing hysteroscopic surgery in ORS were searched in hospital records during the period January 2012 until December 2013. During these years, no procedural sedation in an OBS was offered. Patients undergoing OBS surgery were matched with patients who underwent ORS surgery. Cases were matched by procedure type and menopausal status, and only patients classified as ASA I or II were included. The financial department of the hospital made a specification of costs. For this type of (retrospective) study, formal consent of the ethics committee was not required.

Table 1 Procedural indications and exclusion criteria

Hysteroscopic procedure indications

- Diagnostic hysteroscopy

- Resection of fibroids

- Resection of polyps

- Removal of placental remnants

- Synechiolysis with scissors

- Endometrial ablation

Exclusion criteria

- ASA classification III and IV

- High intracranial pressure

- Heart failure

- Pulmonary hypertension

- Sensitivity for medication used

- Expected difficulties with manual ventilation

- Arterial oxygen saturation $<92 \%$ with O2 supply

- Previous major problems with anaesthesia

\section{Setting}

All OBS procedures were performed in a dedicated hysteroscopy room at the gynaecologic outpatient department of the Catharina Hospital in Eindhoven, the Netherlands. The OBS team consisted of a gynaecologist or supervised resident in training a conscious sedation specialist, nurse- and doctors' assistant. All team members were trained in basic or advanced life support. A time-out procedure was performed prior to giving anaesthesia. Procedures were performed in accordance with current Dutch guidelines on procedural sedation [7]. Shortly after the procedure, patients were conscious and able to make a transfer to a recovery bed. During recovery, a general nurse monitored the patients using the Aldrete scoring system (Table 2). Patients were discharged $60 \mathrm{~min}$ after the last administration of sedatives, when having normal vital parameters and being fully conscious. Instructions concerning physical activity and general hospital discharge instructions were given just prior to leaving the facility, whereas all patients received a phone call on the following day.

During ORS procedures, routine anaesthesia was given; the surgeon was either a gynaecologist or a supervised resident in training.

\section{Medication}

All OBS patients received $1000 \mathrm{mg}$ acetaminophen (paracetamol) and $500 \mathrm{mg}$ naproxen (naproxen) a night and $1 \mathrm{~h}$ before the procedure. During the procedure, patients were moderate to deeply sedated with propofol (Diprivan $®$, AstraZeneca BV, Zoetermeer, the Netherlands) and alfentanyl (Rapifen ${ }^{\circledR}$ Janssen-Cilag BV, Tilburg, the Netherlands) according to the level of sedation stated by the American Society of Anesthesiologists [8]. Granisetron (Kytril ${ }^{\circledR}$ Roche Nederland BV, Woerden, the Netherlands) was administered to prevent nausea. No paracervical, intracervical or intrauterine anaesthesia or antibiotics were given.

In the ORS group, general anaesthesia was mostly induced with propofol (1.5-2.5 mg/kg, Diprivan ${ }^{\circledR}$, AstraZeneca BV, Zoetermeer, the Netherlands) and sufentanil $(0.5-5.0 \mu \mathrm{g} / \mathrm{kg}$, Sufenta ${ }^{\circledR}$, Janssen-Cilag BV, Tilburg, the Netherlands), sometimes a combination of propofol and fentanyl $(1-2 \mathrm{mg} / \mathrm{kg}$, Fentanyl®, Janssen-Cilag BV, Tilburg, the Netherlands), and a laryngeal mask was applied. Loco-regional anaesthesia was supplied by spinal anaesthesia with either bupivacaine (Marcaine ${ }^{\circledR}$ AstraZeneca BV, Zoetermeer, the Netherlands) or lidocaine (Xylocaine ${ }^{\circledR}$, AstraZeneca BV, Zoetermeer, the Netherlands).

\section{Procedures}

When necessary, the cervix was dilated with Hegar dilators. There was no cervical preparation with misoprostol. The 
Table 2 Modified Aldrete score

\begin{tabular}{lll}
\hline Parameter & Description of patient & Score \\
\hline Activity level & Moves all extremities voluntarily on comment & 2 \\
& Moves 2 extremities & 1 \\
Respirations & Cannot move extremities & 0 \\
& Breathes deeply and coughs freely & 2 \\
Circulation & Is dyspneic with shallow limited breathing & 1 \\
& Is apneic & 0 \\
Consciousness & Is 20 mmHg $\leq$ preanesthetic level & 2 \\
& Is $20-50$ mmHg preanesthetic level & 1 \\
Oxygen saturation as determined by pulse & Is 50 mmHg preanesthetic level & 0 \\
oximetry & Is fully awake & 2 \\
& Is arousable on calling & 1 \\
& Is not responding & 0 \\
& Has level $>90 \%$ when breathing room air & 2 \\
\hline
\end{tabular}

Aldrete JA: The post-anesthesia recovery score revisited. J Clin Anesth 1995, 7:89-91

Truclear 5.0 hysteroscopic morcellation system (Smith \& Nephew, Andover, MA, USA) was used for the resection of polyps and small placental remnants. The Truclear 8.0 system was used to morcellate myomas and large polyps or placental remnants. The Novasure system (Hologic, Marlborough, MA, USA) and Thermablate system (Idoman, Killateeaun, Tourmakeady, County Mayo, Ireland) were used for endometrial ablation in OBS. The Thermablate system (Idoman, Killateeaun, Tourmakeady, County Mayo, Ireland) or Cavatherm (Pelvitec, Capelle aan den IJssel, the Netherlands) were used for endometrial ablation in ORS. The Bettochi $4 \mathrm{~mm}$ hysteroscope (Karl Storz, Tuttlingen, Germany) or $3.8 \mathrm{~mm}$ Compact hysteroscope (Richard Wolf, Knittlingen, Germany) were used for the resection of uterine synechia. The Endoflow II pump (Socomed, Lyon, France) or the hysteroscopic fluid management system of Smith \& Nephew (Andover, MA, USA) was used to create distension of the uterine cavity, the latter was also used to monitor intravasation. Intrauterine pressure was aimed at between 80 and $100 \mathrm{mmHg}$ during all hysteroscopic procedures.

\section{Outcomes}

Primary outcome measurements were operation time, admission time, the number of complete performed procedures, need for reoperation and surgical and anaesthetic complication rate. Operation time was defined as the time period between introducing the first instrument until the moment of removing the last instrument. A numeric pain rating scale (NRS) was presented to all patients undergoing office-based procedures. They reported NRS scores before the procedure, during the procedure, after the procedure, when being discharged and during menstruation. In ORS, a pain score was often reported within the first hours after the procedure. All procedures were reviewed retrospectively. Statistical analysis was performed with SPSS 21.0 (IBM, Armonk, New York), using MannWhitney $U$ and chi-square tests.

\section{Results}

A total of 258 procedures were analysed, 129 patients in both groups. An overview of all different types of hysteroscopic procedures performed is shown in Fig. 1. Baseline characteristics between the two groups were not significantly different (Table 3). All OBS procedures were performed under sedation and analgesia with the combination of propofol and alfentanyl (Table 4). Different anaesthetic medications were used in ORS patients. The combination of propofol and sufentanil was the most common administered in ORS (53.1\%). Operation and admission time were significantly shorter in the OBS group (respectively, 11 versus $20 \mathrm{~min}$ and 135 versus $455 \mathrm{~min}$, both $p<0.01)$. The number of incomplete procedures was not different between the two groups, respectively, $3.9 \%$ in OBS group versus $2.3 \%$ in ORS ( $p=0.473)$. In one patient $(0.7 \%)$, the OBS procedure was cancelled due to not being able to effectuate full muscle relaxation. More reoperations were performed in the ORS group compared to the OBS group $(18.6 \%$ versus $7.0 \% ; p<0.01)$. Surgical complications were not significantly different $(1.6 \%$ in both OBS and ORS group; $p=0.652$ ). The complications were grade 1 and 2 according to the Clavien-Dindo system [9], and existed of postoperative infections with need for antibiotic treatment $(n=2)$ in OBS group and uterine perforations $(n=2)$ in the ORS group. All 
Fig. 1 Surgical procedures

\section{Type of hysteroscopic operation}

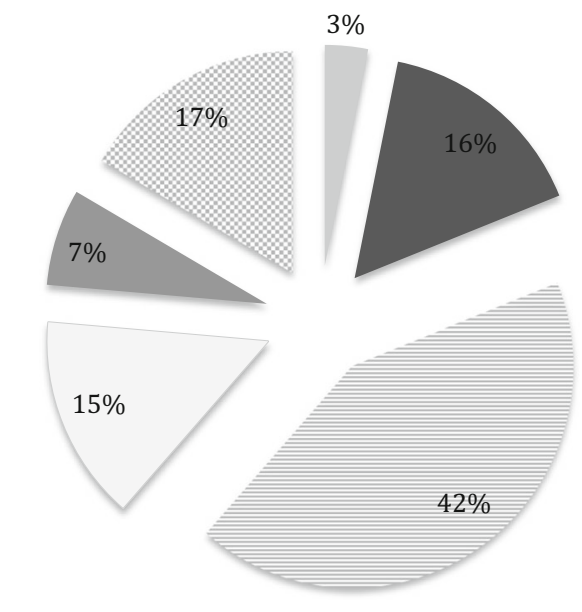

Synechiolysis with scissors

- Diagnostic hysteroscopy

$\equiv$ Morcellation of polyps

Morcellation of myomas

- Morcellation of pregnancy remnant

s. Endometrial ablation

anaesthetic complications were minor. In the OBS group there were airway obstructions with the need of a Mayo tube or positioning manoeuvres (e.g. chin lift or jaw trust) in $3.1 \%$ of patients $(n=4)$. The ORS group had anaesthetic complications in $2.3 \%$ of patients. Analysis revealed two case of postspinal headache, in which one patient needed a blood patch treatment. There was one case of perioperative bradycardia in the ORS group, which was treated with adrenalin. The overall complication rate was $4.7 \%$ in OBS and $3.9 \%$ in ORS $(p=0.758)$.

Median pain score (NRS) before the operation was 0 ( 0 in $87 \%$ of responders, range $0-8)$, during the operation 0 (0 in $99.1 \%$ of responders, range $0-2$ ), after the operation 1 ( 0 or 1 in $68.1 \%$ of responders, range $0-8)$, and during menstruation 4 ( 0 in $19.8 \%$ of responders, range $0-10)$. Financial analysis showed that low or intermediate complex hysteroscopic procedures in an office-based setting are at least half of the costs compared to a clinical setting.

\section{Discussion}

A surgical procedure needs to be performed in a safe setting, well tolerated by patients and appropriate in its goal. If changing the anaesthetic setting, the quality of the surgical procedure and the patient's convenience needs to remain unaltered. Historically, operation rooms were chosen to perform hysteroscopic procedures. Office-based procedures under procedural sedation an analgesia are only recently introduced in gynaecologic practice. Therefore, literature is scarce and data on safety, satisfaction and efficacy among patients are mostly unknown. Our study gives more insight in these office-based procedures.

The presented data show a significant shorter operation and admission time in favour of OBS procedures. This underlines the inefficacy of using operation theatres for relatively short procedures. The admission time in ORS seems quite long. However, the time between admission and actual surgery can vary due to logistics reasons. For example, the surgeon himself discharges patients after completing the operating program. Furthermore, general and spinal anaesthesia can lead to longer postoperative recovery time due to nausea and the inability for spontaneous micturition. The presented data are according to the results of Majholm et al. [10]. This Danish group compared a group of patients with total intravenous anaesthesia with a cohort of monitored anaesthesia care (remifentanyl and paracervical block) during day-surgery operative hysteroscopy and found that the time saving potential is not realized in the operating theatre but rather during the stay in the recovery room [10]. However, there are limitations

Table 3 Baseline characteristics

\begin{tabular}{lccc}
\hline Characteristic & OBS $(n=129)$ & ORS $(n=129)$ & Significance \\
\hline Age & 45 (range 18-78) & 45 (range 23-83) & $p=0.733$ \\
Parity & 2 (range 0-5) & 2 (range 0-8) & $p=0.831$ \\
Amount of vaginal deliveries & 1 (range 0-5) & 1 (range 0-8) & $p=0.896$ \\
Body mass index (BMI) & 24.2 (range 17.7-41.3) & 24.8 (range 17.4-45.5) & $p=0.199$ \\
\hline
\end{tabular}


Table 4 Operation characteristics

\begin{tabular}{llll}
\hline Parameters & OBS $(n=129)$ & ORS $(n=129)$ & Significance \\
\hline Anaesthetic medication (\%) & & & $p<0.01$ \\
- Propofol with (a)fentanyl & 100 & 5.5 & \\
- Propofol with sufentanil & 0 & 53.1 & \\
- Spinal with bupivacaine & 0 & 14.8 & \\
- Spinal with lidocaine & 0 & 17.2 & \\
- Other & 0 & 9.4 & \\
Instrument size (\%) & & & \\
- no report of, or no scope used & 4.7 & 10.0 & \\
- 2.9 mm & 24.0 & 7.8 & \\
- 5 mm & 55.0 & 16.3 & \\
- 8 mm & 16.3 & 65.9 & $p=0.473$ \\
Incomplete procedures (\%) & 3.9 & 2.3 & $p<0.01$ \\
Reoperation (\%) & 7.0 & 18.6 & $p=0.652$ \\
Surgical complications (\%) & 1.6 & 1.6 & $p=0.702$ \\
Anaesthetic complications (\%) & 3.1 & 2.3 & $p=0.758$ \\
Overall complication rate (\%) & 4.7 & 3.9 & $p<0.01$ \\
Operation time (min) & 11 (range 1-37) & $20($ range 2-73) & $p<0.01$ \\
Admission time (min) & 135 (range 60-510) & 455 (range 240-2865) & $p=0.466$ \\
Pain score after (0-10) & 1 (range 0-8) & 0 (range 0-7) & \\
\hline
\end{tabular}

of office-based hysteroscopic procedures. In general, patients will have doubts considering office-based procedures, due to fear of pain and discomfort during the procedure. Data show that none of the procedures in the OBS group were cancelled due to discomfort and NRS did not differ significantly postoperatively between the two groups. In OBS patients the NRS before, during and after the procedure showed acceptable, and even low, pain scores. These data suggest that office-based surgery is comparable to working in an operation theatre. However, the pain ratings were not fully comparable between the two groups due to the great variety in time of recording after the operation in the ORS group. Centini et al. compared parenterally administered moderate sedation and paracervical block versus general anaesthesia during day-case hysteroscopy for polypectomy [11]. Analysis of patients' discomfort showed a higher frequency of movements and moans in the paracervical block and sedation group and more often use of analgesic or antiemetic drugs after surgery in the general anaesthesia group, although both findings were not statistically significant. In the ORS the instruments used had a significantly larger diameter. The size of the instrument, or size of the intrauterine abnormality, did not influence the setting of the operation.

A second reason to perform surgery in an inpatient setting is the risk of possible occurrence of complications and the inadequacy of the circumstances to deal with them. The data demonstrate that surgical complications appeared in only $1.6 \%$ of the OBS group (grade 1 or 2 postoperative infections). A study by Wortman et al. [12] reported a complication rate of $2.7 \%$ for hysteroscopic procedures in OBS, which is comparable with our data. We found four anaesthetic complications in the OBS group and three in the ORS group which was not statistically significantly different. All reported complications in the OBS group were airway obstructions with the need for a mayo tube or positioning manoeuvres (e.g. chin lift or jaw trust), being also the most reported complication in the literature of the combined alfentanil and propofol [13]. There was no use of naloxone. In the ORS group most complications were due to spinal anaesthesia $(n=2)$.

The amount of incomplete procedures was $3.9 \%$ in the OBS group $(n=5)$ and not significantly different compared to the ORS group $(2.3 \%, n=4)$. Success rates of office-based hysteroscopic procedures such as polypectomy have been reported to be around $73 \%$ after 2 years. [14]. Patient discomfort and large fluid deficit due to longer resection time are found to result in partial or failed removal $[14,15]$. The weakness of our study is the non-randomized study design. However, all included patients received diagnostic hysteroscopy prior to hysteroscopic surgery. Every week during this pilot period, a limited number of patients were offered the opportunity of conscious sedation surgery. If planning of a patient for sedation was not possible within 2 weeks following diagnostic hysteroscopy, an inpatient procedure was offered. During the pilot period a total of 237 patients had hysteroscopic surgery in ORS compared to 129 in an inpatient setting. There were no differences in patient characteristics between the in and outpatient group in this period, all patients were classificated as ASA 1 or 2. 
The significant shorter admission and operating time could lead to substantial cost savings as is already shown by the estimation of the financial department of our hospital. Other advantages mentioned in the literature, are the ability to combine the diagnostic and treatment phase of patient management without the need for multiple interventions and anaesthetics. Furthermore, women prefer the familiar environment of the office setting and the physician benefits from the more efficient use of time and ease of scheduling [12].

\section{Conclusion}

Overall, office-based hysteroscopic procedures with the use of sedation and analgesia are safe, with a low complication rate, not painful and lead to substantially shorter operation and admission times. Office-based hysteroscopic procedures could lead to lower healthcare costs. Cost-effectiveness analysis and randomized controlled trials should be performed to give a definite answer on this topic.

Author's contribution R.M. Smits: data collection and management, data analysis, manuscript writing

N.P.M. Kuijsters: data collecting, data analysis

L. Braam: protocol and project development, manuscript editing

H.A.A.M. van Vliet: protocol and project development, manuscript editing

B.C. Schoot: protocol and project development, manuscript editing

\section{Compliance with ethical standards}

Conflict of interest Author van Vliet received fees on hourly basis for lectures and training on hysteroscopic morcellation by Smith and Nephew. The fees were donated to a foundation which promotes research in obstetrics and gynaecology. Author Smits, Author Kuijsters, Author Braam, and Author Schoot declare that they have no conflict of interest.

Ethical approval Our study was exempt for Institutional Review Board (IRB) approval as the use of existing data was provided without the identification of living individuals. Our research did not involve animals.

\section{References}

1. Emanuel MH (2013) New developments in hysteroscopy. Best Pract Res Clin Obstet Gynaecol 27(3):421-429

2. Di Spiezio SA, Bettocchi S, Spinelli M, Huida M, Nappi L, Angioni S, Sosa Fernandez LM, Nappi C (2010) Review of new office-based hysteroscopic procedures 2003-2009. J Minim Invasive Gynecol 17(4):436-448

3. Favilli A, Mazzon I, Gerli S (2015) Pain in office hysteroscopy: it is not just a matter of size. Comment on: Paulo AA, Solheiro MH, Paulo CO. Is pain better tolerated with mini-hysteroscopy than with conventional device? A systematic review and meta-analysis: hysteroscopy scope size and pain. Arch Gynecol Obstet 292:987-994

4. Cicinelli E (2010) Hysteroscopy without anesthesia: review of recent literature. J Minim Invasive Gynecol 17(6):703-708

5. Lindheim SR, Kavic S, Shulman SV, Sauer MV (2000) Operative hysteroscopy in the office setting. J Am Assoc Gynecol Laparosc 7:65-69

6. Marsh F, Kremer C, Duffy S (2004) Delivering an effective outpatient service in gynaecology. A randomised controlled trial analysing the cost of outpatient versus daycase hysteroscopy. BJOG 111:243-248

7. Dutch Institute for Healthcare Improvement CBO (2009) Guideline PSA at locations outside the operation room

8. American Society of Anesthesiologists (2014) Continuum of Depth of Sedation: Definition of general anesthesia and levels of sedation/ analgesia

9. Clavien PA, Barkun J, de Oliveira ML, Vauthey JN, Dindo D, Schulick RD, de Santibañes E, Pekolj J, Slankamenac K, Bassi C, Graf R, Vonlanthen R, Padbury R, Cameron JL, Makuuchi M (2009) The Clavien-Dindo classification of surgical complications: five-year experience. Ann Surg 250(2):187-196

10. Majholm B, Bartholdy J, Clausen HV, Virkus RA, Engbaek J, Moller AM (2012) Comparison between local anaesthesia with remifentanil and total intravenous anaesthesia for operative hysteroscopic procedures in day surgery. Br J Anaesth 108:245-253

11. Centini G, Calonaci A, Lazzeri L, Tosti C, Palomba C, Puzzutiello R, Luisi S, Petraglia F, Zupi E (2015) Parenterally administered moderate sedation and paracervical block versus general anesthesia for hysteroscopic polypectomy: a pilot study comparing postoperative outcomes. J Minim Invasive Gynecol 22:193-198

12. Wortman M, Dagget A, Ball C (2013) Operative hysteroscopy in an office-based surgical setting: review of patient safety and satisfaction in 414 cases. J Minim Invasive Gynecol 20:56-63

13. Nilsson A, Nilsson L, Ustaal E, Sjoberg F (2012) Alfentanil and patient-controlled propofol sedation facilitate gynaecological outpatient surgery with increased risk of respiratory events. Acta Anaesthesiol Scand 56:1123-1129

14. Cooper NA, Clark TJ, Middleton L, Diwakar L, Smith P, Denny E, Roberts T, Stobert L, Jowett S, Daniels J (2015) OPT Trial Collaborative Group. Outpatient versus inpatient uterine polyp treatment for abnormal uterine bleeding: randomised controlled non-inferiority study. BMJ: 350

15. Rubino RJ, Lukes AS (2015) Twelve-month outcomes for patients undergoing hysteroscopic morcellation of uterine polyps and myomas in an office or ambulatory surgical center. J Minim Invasive Gynecol 22:285-290 